\title{
CHARACTERIZATIONS OF JORDAN DERIVATIONS ON STRONGLY DOUBLE TRIANGLE SUBSPACE LATTICE ALGEBRAS
}

\author{
YUN-HE CHEN and JIAN-KUI LI ${ }^{凶}$
}

(Received 6 December 2010)

\begin{abstract}
Let $\mathcal{D}$ be a strongly double triangle subspace lattice on a nonzero complex reflexive Banach space $X$ and let $\delta: \operatorname{Alg} \mathcal{D} \rightarrow \operatorname{Alg} \mathcal{D}$ be a linear mapping. We show that $\delta$ is Jordan derivable at zero, that is, $\delta(A B+B A)=\delta(A) B+A \delta(B)+\delta(B) A+B \delta(A)$ whenever $A B+B A=0$ if and only if $\delta$ has the form $\delta(A)=\tau(A)+\lambda A$ for some derivation $\tau$ and some scalar $\lambda$. We also show that if the dimension of $X$ is greater than 2, then $\delta$ satisfies $\delta(A B+B A)=\delta(A) B+A \delta(B)+\delta(B) A+B \delta(A)$ whenever $A B=0$ if and only if $\delta$ is a derivation.
\end{abstract}

2010 Mathematics subject classification: primary 47B47.

Keywords and phrases: derivation, Jordan derivation, subspace lattice.

\section{Introduction}

Throughout this paper, $X$ will denote a nonzero complex reflexive Banach space with topological dual $X^{*}$. The terms operator and subspace will mean 'bounded linear mapping' and 'norm closed linear manifold', respectively. As usual, the set of all bounded linear operators on $X$ is denoted by $B(X)$. If $e^{*} \in X^{*}$ and $f \in X$, then $e^{*} \otimes f$ denotes the operator $\left(e^{*} \otimes f\right) x=e^{*}(x) f$ for every $x \in X$. For any nonempty subset $Y \subseteq X, Y^{\perp}$ denotes its annihilator, that is, $Y^{\perp}=\left\{f^{*} \in X^{*}: f^{*}(y)=0\right.$ for every $\left.y \in Y\right\}$. For any nonempty subset $Z \subseteq X^{*},{ }^{\perp} Z$ denotes its pre-annihilator, that is, ${ }^{\perp} Z=\{x \in X$ : $f^{*}(x)=0$ for every $f^{*} \in Z$ \}.

By a subspace lattice on $X$ we mean a family $\mathcal{L}$ of subspaces of $X$ with (0) and $X$ in $\mathcal{L}$ such that for every family $\left\{L_{\gamma}\right\}_{\gamma \in \Gamma}$ of elements of $\mathcal{L}$, both $\bigcap_{\gamma \in \Gamma} L_{\gamma}$ and $\bigvee_{\gamma \in \Gamma} L_{\gamma}$ belong to $\mathcal{L}$, where $\vee$ denotes 'closed linear span'. For any subspace lattice $\mathcal{L}$ on $X$, we define $\operatorname{Alg} \mathcal{L}$ by

$$
\operatorname{Alg} \mathcal{L}=\{T \in B(X): T L \subseteq L, \text { for every } L \in \mathcal{L}\}
$$

and $\mathcal{L}^{\perp}=\left\{L^{\perp}: L \in \mathcal{L}\right\}$.

This work is supported by NSF of China.

(C) 2011 Australian Mathematical Publishing Association Inc. 0004-9727/2011 \$16.00 
A double triangle subspace lattice on $X$ is a set $\mathcal{D}=\{(0), K, L, M, X\}$ of subspaces of $X$ satisfying $K \cap L=L \cap M=M \cap K=(0)$ and $K \vee L=L \vee M=M \vee$ $K=X$ (see $[2,6,8])$. If one of the three sums $K+L, L+M$ and $M+K$ is closed, we say that $\mathcal{D}$ is a strongly double triangle subspace lattice. It is known from [7, Proposition 3.1] that $\operatorname{Alg} \mathcal{D}$ contains no rank-one operators. Observe that $\mathcal{D}^{\perp}=$ $\left\{(0), K^{\perp}, L^{\perp}, M^{\perp}, X^{*}\right\}$ is a double triangle subspace lattice on the reflexive Banach space $X^{*}$. We follow the notation used in [6, Definition 2.1] and put $K_{0}=K \cap(L+M)$, $L_{0}=L \cap(M+K), M_{0}=M \cap(K+L)$ and $K_{p}=K^{\perp} \cap\left(L^{\perp}+M^{\perp}\right), L_{p}=L^{\perp} \cap\left(M^{\perp}+\right.$ $\left.K^{\perp}\right), M_{p}=M^{\perp} \cap\left(K^{\perp}+L^{\perp}\right)$. Note that $K_{p}, L_{p}$ and $M_{p}$ play the same role for $\mathcal{D}^{\perp}$ as $K_{0}, L_{0}$ and $M_{0}$ do for $\mathcal{D}$. By [6, Lemma 2.2], the dimensions of the linear manifolds $K_{0}, L_{0}$ and $M_{0}$ are the same and the common dimension is denoted by $m$. Similarly, the dimensions of the linear manifolds $K_{p}, L_{p}$ and $M_{p}$ are the same and the common dimension is denoted by $n$.

Let $\mathcal{A}$ be a unital algebra. Recall that a linear mapping $\delta$ from $\mathcal{A}$ into itself is a derivation (respectively, a generalized derivation) if $\delta(A B)=\delta(A) B+A \delta(B)$ (respectively, $\delta(A B)=\delta(A) B+A \delta(B)-A \delta(I) B)$ for any $A, B \in \mathcal{A}$. Recall that $\delta$ is derivable at $Z \in \mathcal{A}$ if $\delta(A B)=\delta(A) B+A \delta(B)$ for any $A, B \in \mathcal{A}$ with $A B=Z$, and $\delta$ is Jordan derivable at $Z \in \mathcal{A}$ if $\delta(A B+B A)=\delta(A) B+A \delta(B)+\delta(B) A+B \delta(A)$ for any $A, B \in \mathcal{A}$ with $A B+B A=Z$.

In recent years, there have been a number of papers on the study of conditions under which derivations and Jordan derivations of operator algebras can be completely determined by the action on some sets of operators (for example, see $[1,3,4,9,10]$ ). In [9], Pang and Yang showed that every linear mapping $\delta$ which is derivable at zero on a strongly double triangle subspace lattice algebra has the form $\delta(A)=\tau(A)+\lambda A$ for some derivation $\tau$ and some scalar $\lambda$. Motivated by this, we study the local action of Jordan derivations on $\operatorname{Alg} \mathcal{D}$ for a strongly double triangle subspace lattice $\mathcal{D}$. Our main results are Theorems 2.1 and 2.5. It is shown that every linear mapping $\delta$ which is Jordan derivable at zero from $\operatorname{Alg} \mathcal{D}$ into itself has the form $\delta(A)=\tau(A)+\lambda A$ for some derivation $\tau$ and some scalar $\lambda$. We also show that if the dimension of $X$ is greater than two, then every linear mapping $\delta$ from $\operatorname{Alg} \mathcal{D}$ into itself satisfying $\delta(A B+B A)=\delta(A) B+A \delta(B)+\delta(B) A+B \delta(A)$ whenever $A B=0$ is a derivation. We first recall some results which we require in Section 2.

Lemma 1.1 [6, Lemma 2.1]. Let $\mathcal{D}$ be a double triangle subspace lattice on $X$. Then the following statements hold:

(i) $K_{0} \subseteq K \subseteq^{\perp} K_{p}, L_{0} \subseteq L \subseteq^{\perp} L_{p}$ and $M_{0} \subseteq M \subseteq^{\perp} M_{p}$;

(ii) $K_{0} \cap L_{0}=L_{0} \cap M_{0}=M_{0} \cap K_{0}=(0)$;

(iii) $K_{p} \cap L_{p}=L_{p} \cap M_{p}=M_{p} \cap K_{p}=(0)$;

(iv) $K_{0}+L_{0}=L_{0}+M_{0}=M_{0}+K_{0}=K_{0}+L_{0}+M_{0}$;

(v) $K_{p}+L_{p}=L_{p}+M_{p}=M_{p}+K_{p}=K_{p}+L_{p}+M_{p}$.

Theorem 1.2 [6, Theorem 2.1]. Let $\mathcal{D}$ be a double triangle subspace lattice on $X$.

(i) Every finite-rank operator of $\mathrm{Alg} \mathcal{D}$ has even rank (possibly zero). 
(ii) If $e, f \in X$ and $e^{*}, f^{*} \in X^{*}$ are nonzero vectors satisfying $e \in K_{0}, f \in L_{0}, e+f \in$ $M_{0}$ and $e^{*} \in K_{p}, f^{*} \in L_{p}, e^{*}+f^{*} \in M_{p}$, then $R=e^{*} \otimes f-f^{*} \otimes e$ is a rank-two operator of Alg $\mathcal{D}$. Moreover, every rank-two operator of $\mathrm{Alg} \mathcal{D}$ has this form for some such vectors $e, f, e^{*}, f^{*}$.

(iii) Every nonzero finite-rank operator of $\operatorname{Alg} \mathcal{D}$ (if there are any) is a finite sum of rank-two operators of $\mathrm{Alg} \mathcal{D}$.

(iv) $\operatorname{Alg} \mathcal{D}$ contains a nonzero finite-rank operator if and only if $m \neq 0$ and $n \neq 0$.

Lemma 1.3 [6, Lemma 3.2]. Let $\mathcal{D}$ be a double triangle subspace lattice on $X$. Let $e, f, e^{*}$ and $f^{*}$ be nonzero vectors satisfying $e \in K_{0}, f \in L_{0}, e+f \in M_{0}, e^{*} \in K_{p}$, $f^{*} \in L_{p}$ and $e^{*}+f^{*} \in M_{p}$ and put $R=e^{*} \otimes f-f^{*} \otimes e$. Then $e^{*}(f)=-f^{*}(e)$ and $R^{2}=e^{*}(f) R$.

Theorem 1.4 [6, Theorem 2.3]. Let $\mathcal{D}$ be a double triangle subspace lattice on $X$. If the vector sum $K+L$ is closed, then:

(i) $K_{0}$ is dense in $K, L_{0}$ is dense in $L$ and $M_{0}=M$;

(ii) $K_{0}+L_{0}+M_{0}$ is dense in $X$;

(iii) $K_{p}+L_{p}+M_{p}$ is dense in $X^{*}$.

The following lemma is essentially included in the proof of [9, Theorem 2.3].

Lemma 1.5. Let $\mathcal{D}$ be a strongly double triangle subspace lattice on $X$. Then every rank-two operator is a linear combination of at most two rank-two idempotents in $\operatorname{Alg} \mathcal{D}$.

\section{Main results}

Our first result is Theorem 2.1 which says that every linear mapping Jordan derivable at zero on a strongly double triangle subspace lattice algebra is a special kind of generalized derivation.

Theorem 2.1. Let $\mathcal{D}$ be a strongly double triangle subspace lattice on $X$ and let $\delta: \operatorname{Alg} \mathcal{D} \rightarrow \operatorname{Alg} \mathcal{D}$ be a linear mapping. If $\delta$ is Jordan derivable at zero, then $\delta(I)=\lambda I$ for some scalar $\lambda \in \mathbb{C}$, and there is a derivation $\tau$ such that $\delta(A)=\tau(A)+\lambda A$ for every $A \in \mathrm{Alg} \mathcal{D}$. In particular, if $\delta(I)=0$, then $\delta$ is a derivation.

To prove Theorem 2.1, we need some lemmas. The first of the following lemmas is included in the proof of [3, Theorem 3.1]. We leave the proof to the reader.

Lemma 2.2. If $\delta$ is Jordan derivable at zero from a unital algebra $\mathcal{A}$ into itself and $\delta(I)=0$, then for any idempotents $P$ and $Q$ in $\mathcal{A}$, the following statements hold:

(i) $\delta(P)=\delta(P) P+P \delta(P)$;

(ii) $\delta(P Q+Q P)=\delta(P) Q+P \delta(Q)+\delta(Q) P+Q \delta(P)$.

For every $A \in \operatorname{Alg} \mathcal{D}$ and every rank-two operator $R \in \operatorname{Alg} \mathcal{D}$, the operator $A R$ (respectively, $R A$ ) has rank at most two, so since $A l g \mathcal{D}$ contains no rank-one operators, it is zero or has rank two. 
Lemma 2.3. Let $\mathcal{D}$ be a strongly double triangle subspace lattice on $X$. If $\delta$ is Jordan derivable at zero from $\operatorname{Alg} \mathcal{D}$ into itself and $\delta(I)=0$, then for every $A \in A \lg \mathcal{D}$ and every rank-two operator $R \in A \lg \mathcal{D}$, we have $\delta(A R+R A)=\delta(A) R+A \delta(R)+\delta(R) A+$ $R \delta(A)$.

Proof. Combining Lemmas 1.5 and 2.2, for any rank-two operators $R_{1}, R_{2} \in \operatorname{Alg} \mathcal{D}$, we have $\delta\left(R_{1} R_{2}+R_{2} R_{1}\right)=\delta\left(R_{1}\right) R_{2}+R_{1} \delta\left(R_{2}\right)+\delta\left(R_{2}\right) R_{1}+R_{2} \delta\left(R_{1}\right)$. For every $A \in$ Alg $\mathcal{D}$ and every rank-two idempotent $\tilde{R} \in \operatorname{Alg} \mathcal{D}$, since $\tilde{R}^{\perp} A \tilde{R}^{\perp} \tilde{R}+\tilde{R} \tilde{R}^{\perp} A \tilde{R}^{\perp}=0$, by assumption we have

$$
\begin{aligned}
& \delta\left(\tilde{R}^{\perp} A \tilde{R}^{\perp} \tilde{R}+\tilde{R} \tilde{R}^{\perp} A \tilde{R}^{\perp}\right) \\
& \quad=\delta\left(\tilde{R}^{\perp} A \tilde{R}^{\perp}\right) \tilde{R}+\tilde{R}^{\perp} A \tilde{R}^{\perp} \delta(\tilde{R})+\delta(\tilde{R}) \tilde{R}^{\perp} A \tilde{R}^{\perp}+\tilde{R} \delta\left(\tilde{R}^{\perp} A \tilde{R}^{\perp}\right) .
\end{aligned}
$$

Since $\tilde{R} A$ is zero or a rank-two operator in $\operatorname{Alg} \mathcal{D}$, it follows that

$$
\delta(\tilde{R} A \tilde{R}+\tilde{R} \tilde{R} A)=\delta(\tilde{R} A) \tilde{R}+\tilde{R} A \delta(\tilde{R})+\delta(\tilde{R}) \tilde{R} A+\tilde{R} \delta(\tilde{R} A) .
$$

Similarly, we have

$$
\delta\left(\tilde{R}^{\perp} A \tilde{R} \tilde{R}+\tilde{R} \tilde{R}^{\perp} A \tilde{R}\right)=\delta\left(\tilde{R}^{\perp} A \tilde{R}\right) \tilde{R}+\tilde{R}^{\perp} A \tilde{R} \delta(\tilde{R})+\delta(\tilde{R}) \tilde{R}^{\perp} A \tilde{R}+\tilde{R} \delta\left(\tilde{R}^{\perp} A \tilde{R}\right) .
$$

Since $A=\tilde{R}^{\perp} A \tilde{R}^{\perp}+\tilde{R} A+\tilde{R}^{\perp} A \tilde{R}$, it follows from (2.1)-(2.3) that $\delta(A \tilde{R}+\tilde{R} A)=$ $\delta(A) \tilde{R}+A \delta(\tilde{R})+\delta(\tilde{R}) A+\tilde{R} \delta(A)$. Hence by Lemma 1.5 , for every rank-two operator $R \in \operatorname{Alg} \mathcal{D}$, we have $\delta(A R+R A)=\delta(A) R+A \delta(R)+\delta(R) A+R \delta(A)$.

For a double triangle subspace lattice, each $x$ in $K_{0}$ can be expressed uniquely in the form $x_{1}+x_{2}$, where $x_{1} \in L_{0}$ and $x_{2} \in M_{0}$. Similarly, each $f^{*}$ in $K_{p}$ can be expressed uniquely in the form $f_{1}^{*}+f_{2}^{*}$, where $f_{1}^{*} \in L_{p}$ and $f_{2}^{*} \in M_{p}$.

Lemma 2.4. Suppose that $\mathcal{D}$ is a strongly double triangle subspace lattice on $X$ with $K+L=X$. Let $\Phi: K_{0} \times K_{p} \rightarrow \operatorname{Alg} \mathcal{D}$ be a bilinear mapping. If $\Phi\left(x, f^{*}\right)\left(\operatorname{ker}\left(f^{*}\right) \cap\right.$ $\left.\operatorname{ker}\left(f_{1}^{*}\right)\right) \subseteq \operatorname{span}\left\{x, x_{1}\right\}$, for every $x=x_{1}+x_{2} \in K_{0}$ and every $f^{*}=f_{1}^{*}+f_{2}^{*} \in K_{p}$, where $x_{1} \in L_{0}, x_{2} \in M_{0}, f_{1}^{*} \in L_{p}$ and $f_{2}^{*} \in M_{p}$, then there exist linear mappings $S: L_{0} \rightarrow L_{0}$, $T: K_{0} \rightarrow K_{0}, V: K_{p} \rightarrow X^{*}$ and $W: K_{p} \rightarrow X^{*}$ such that

$$
\Phi\left(x, f^{*}\right)=f^{*} \otimes S x_{1}+f_{1}^{*} \otimes T x+V f^{*} \otimes x+W f^{*} \otimes x_{1},
$$

for every $x=x_{1}+x_{2} \in K_{0}$ and every $f^{*}=f_{1}^{*}+f_{2}^{*} \in K_{p}$.

Proof. For any nonzero vectors $x \in K_{0}$ and $f^{*} \in K_{p}$, since $\Phi\left(x, f^{*}\right)\left(\operatorname{ker}\left(f^{*}\right) \cap\right.$ $\left.\operatorname{ker}\left(f_{1}^{*}\right)\right) \subseteq \operatorname{span}\left\{x, x_{1}\right\}$, there exist linear functionals $V_{x, f^{*}}$ and $W_{x, f^{*}}$ on $\operatorname{ker}\left(f^{*}\right) \cap$ $\operatorname{ker}\left(f_{1}^{*}\right)$ such that for every $z \in \operatorname{ker}\left(f^{*}\right) \cap \operatorname{ker}\left(f_{1}^{*}\right)$,

$$
\Phi\left(x, f^{*}\right) z=V_{x, f^{*}}(z) x+W_{x, f^{*}}(z) x_{1} .
$$

Since $K+L$ is closed and $K \cap L=(0)$, we have $V_{x, f^{*}}$ and $W_{x, f^{*}}$ are continuous by [5, Corollary 1.8.8].

Let $\omega_{f^{*}}$ be in $L_{0}$ such that $f^{*}\left(\omega_{f^{*}}\right)=1$ and $\gamma_{f^{*}}$ be in $K_{0}$ such that $f_{1}^{*}\left(\gamma_{f^{*}}\right)=1$. Then $X=\mathbb{C} \omega_{f^{*}} \oplus \mathbb{C} \gamma_{f^{*}} \oplus\left(\operatorname{ker}\left(f^{*}\right) \cap \operatorname{ker}\left(f_{1}^{*}\right)\right)$. Let $\tilde{V}_{x, f^{*}}$ and $\tilde{W}_{x, f^{*}}$ be continuous 
extensions of $V_{x, f^{*}}$ and $W_{x, f^{*}}$ to $X$. Then $\tilde{V}_{x, f^{*}}-\tilde{V}_{x, f^{*}}\left(\omega_{f^{*}}\right) f^{*}-\tilde{V}_{x, f^{*}}\left(\gamma_{f^{*}}\right) f_{1}^{*}$ and $\tilde{W}_{x, f^{*}}-\tilde{W}_{x, f^{*}}\left(\omega_{f^{*}}\right) f^{*}-\tilde{W}_{x, f^{*}}\left(\gamma_{f^{*}}\right) f_{1}^{*}$ are also continuous extensions of $V_{x, f^{*}}$ and $W_{x, f^{*}}$ and vanish at $\operatorname{span}\left\{\omega_{f^{*}}, \gamma_{f^{*}}\right\}$. We use $V_{x, f^{*}}$ and $W_{x, f^{*}}$ to denote such extensions.

Now define linear mappings $S_{f^{*}}: L_{0} \rightarrow L_{0}$ by $S_{f^{*}} y_{1}=\Phi\left(y, f^{*}\right) \omega_{f^{*}}$ and $T_{f^{*}}: K_{0} \rightarrow$ $K_{0}$ by $T_{f^{*}} y=\Phi\left(y, f^{*}\right) \gamma_{f^{*}}$, for every $y=y_{1}+y_{2} \in K_{0}$, where $y_{1} \in L_{0}$ and $y_{2} \in M_{0}$. Then for $\lambda, \mu \in \mathbb{C}$ and every $z \in \operatorname{ker}\left(f^{*}\right) \cap \operatorname{ker}\left(f_{1}^{*}\right)$,

$$
\begin{aligned}
\Phi\left(x, f^{*}\right)\left(\lambda \omega_{f^{*}}+\mu \gamma_{f^{*}}+z\right)= & \lambda S_{f^{*}} x_{1}+\mu T_{f^{*}} x+V_{x, f^{*}}(z) x+W_{x, f^{*}}(z) x_{1} \\
=f^{*}\left(\lambda \omega_{f^{*}}+\mu \gamma_{f^{*}}+z\right) S_{f^{*}} x_{1}+f_{1}^{*}\left(\lambda \omega_{f^{*}}+\mu \gamma_{f^{*}}+z\right) T_{f^{*}} x & \\
& \quad+V_{x, f^{*}}\left(\lambda \omega_{f^{*}}+\mu \gamma_{f^{*}}+z\right) x+W_{x, f^{*}}\left(\lambda \omega_{f^{*}}+\mu \gamma_{f^{*}}+z\right) x_{1} .
\end{aligned}
$$

Hence

$$
\Phi\left(x, f^{*}\right)=f^{*} \otimes S_{f^{*}} x_{1}+f_{1}^{*} \otimes T_{f^{*}} x+V_{x, f^{*}} \otimes x+W_{x, f^{*}} \otimes x_{1},
$$

for every $x \in K_{0}$ and every $f^{*} \in K_{p}$.

We claim that $V_{x, f^{*}}$ and $W_{x, f^{*}}$ depend only on $f^{*}$. To see this, fix a nonzero functional $f^{*}=f_{1}^{*}+f_{2}^{*} \in K_{p}$, where $f_{1}^{*} \in L_{p}$ and $f_{2}^{*} \in M_{p}$. Let $x=x_{1}+x_{2}$ and $y=$ $y_{1}+y_{2}$ be nonzero vectors in $K_{0}$, where $x_{1}, y_{1} \in L_{0}$ and $x_{2}, y_{2} \in M_{0}$. Then $x+y=$ $\left(x_{1}+y_{1}\right)+\left(x_{2}+y_{2}\right)$, where $x+y \in K_{0}, x_{1}+y_{1} \in L_{0}$ and $x_{2}+y_{2} \in M_{0}$.

Suppose that $x$ and $y$ are linearly independent. Since $K_{0} \cap L_{0}=L_{0} \cap M_{0}=M_{0} \cap$ $K_{0}=(0)$, we have that $x_{1}$ and $y_{1}$ are linearly independent and $x_{2}$ and $y_{2}$ are linearly independent. Then for every $z \in \operatorname{ker}\left(f^{*}\right) \cap \operatorname{ker}\left(f_{1}^{*}\right)$, by (2.4),

$$
\begin{aligned}
& \Phi\left(x+y, f^{*}\right)\left(\omega_{f^{*}}+\gamma_{f^{*}}+z\right) \\
& \quad=S_{f^{*}}\left(x_{1}+y_{1}\right)+T_{f^{*}}(x+y)+V_{x+y, f^{*}}(z)(x+y)+W_{x+y, f^{*}}(z)\left(x_{1}+y_{1}\right)
\end{aligned}
$$

and

$$
\begin{aligned}
\Phi(x & \left.+y, f^{*}\right)\left(\omega_{f^{*}}+\gamma_{f^{*}}+z\right) \\
& =\Phi\left(x, f^{*}\right)\left(\omega_{f^{*}}+\gamma_{f^{*}}+z\right)+\Phi\left(y, f^{*}\right)\left(\omega_{f^{*}}+\gamma_{f^{*}}+z\right) \\
& =S_{f^{*}} x_{1}+T_{f^{*}} x+V_{x, f^{*}}(z) x+W_{x, f^{*}}(z) x_{1}+S_{f^{*}} y_{1}+T_{f^{*}} y+V_{y, f^{*}}(z) y+W_{y, f^{*}}(z) y_{1} .
\end{aligned}
$$

Comparing the above equations,

$$
\begin{aligned}
& \left(V_{x+y, f^{*}}(z)-V_{x, f^{*}}(z)\right) x+\left(V_{x+y, f^{*}}(z)-V_{y, f^{*}}(z)\right) y \\
& \quad=\left(W_{x, f^{*}}(z)-W_{x+y, f^{*}}(z)\right) x_{1}+\left(W_{y, f^{*}}(z)-W_{x+y, f^{*}}(z)\right) y_{1} \in K_{0} \cap L_{0}=(0) .
\end{aligned}
$$

Hence $V_{x+y, f^{*}}=V_{x, f^{*}}=V_{y, f^{*}}$ and $W_{x+y, f^{*}}=W_{x, f^{*}}=W_{y, f^{*}}$.

Suppose that $x$ and $y$ are linearly dependent. Let $y=k x$. Then $y_{1}=k x_{1}$ and $y_{2}=k x_{2}$. By (2.4),

$$
\Phi\left(y, f^{*}\right)=f^{*} \otimes S_{f^{*}}\left(k x_{1}\right)+f_{1}^{*} \otimes T_{f^{*}}(k x)+V_{y, f^{*}} \otimes y+W_{y, f^{*}} \otimes\left(k x_{1}\right)
$$

and

$$
\Phi\left(y, f^{*}\right)=k \Phi\left(x, f^{*}\right)=k f^{*} \otimes S_{f^{*}} x_{1}+k f_{1}^{*} \otimes T_{f^{*}} x+k V_{x, f^{*}} \otimes x+k W_{x, f^{*}} \otimes x_{1},
$$

which yields $\left(V_{y, f^{*}}-V_{x, f^{*}}\right) \otimes y=\left(W_{x, f^{*}}-W_{y, f^{*}}\right) \otimes x_{1}$. It follows from $K_{0} \cap L_{0}=(0)$ that $V_{x, f^{*}}=V_{y, f^{*}}$ and $W_{x, f^{*}}=W_{y, f^{*}}$. We establish the claim. 
Therefore, for every $f^{*} \in K_{p}$, there exist unique functionals $V_{f^{*}}$ and $W_{f^{*}}$ in $X^{*}$ which vanish at $\operatorname{span}\left\{\omega_{f^{*}}, \gamma_{f^{*}}\right\}$ such that

$$
\Phi\left(x, f^{*}\right)=f^{*} \otimes S_{f^{*}} x_{1}+f_{1}^{*} \otimes T_{f^{*}} x+V_{f^{*}} \otimes x+W_{f^{*}} \otimes x_{1},
$$

for every $x \in K_{0}$.

Let $f^{*}=f_{1}^{*}+f_{2}^{*}$ and $g^{*}=g_{1}^{*}+g_{2}^{*}$ be nonzero vectors in $K_{p}$, where $f_{1}^{*}, g_{1}^{*} \in L_{p}$ and $f_{2}^{*}, g_{2}^{*} \in M_{p}$. We claim that if $f^{*}$ and $g^{*}$ are linearly independent, then $S_{g^{*}}-S_{f^{*}}$ is a scalar multiple of the identity $I_{L_{0}}$ on $L_{0}$ and $T_{g^{*}}-T_{f^{*}}$ is a scalar multiple of the identity $I_{K_{0}}$ on $K_{0}$. The independence of $f^{*}$ and $g^{*}$ gives $\operatorname{ker}\left(f^{*}\right) \nsubseteq \operatorname{ker}\left(g^{*}\right)$ and $\operatorname{ker}\left(g^{*}\right) \nsubseteq \operatorname{ker}\left(g^{*}\right)$, so there exist two vectors $u \in \operatorname{ker}\left(f^{*}\right)$ and $v \in \operatorname{ker}\left(g^{*}\right)$ such that $g^{*}(u)=1$ and $f^{*}(v)=1$. For every $x_{1} \in L_{0}$, there exist unique vectors $x \in K_{0}$ and $x_{2} \in M_{0}$ such that $x_{1}=x-x_{2}$. By (2.5),

$$
\Phi\left(x, f^{*}+g^{*}\right)=\left(f^{*}+g^{*}\right) \otimes S_{f^{*}+g^{*}} x_{1}+\left(f_{1}^{*}+g_{1}^{*}\right) \otimes T_{f^{*}+g^{*}} x+V_{f^{*}+g^{*}} \otimes x+W_{f^{*}+g^{*}} \otimes x_{1}
$$

and

$$
\begin{aligned}
& \Phi\left(x, f^{*}+g^{*}\right)= \Phi\left(x, f^{*}\right)+\Phi\left(x, g^{*}\right) \\
&=f^{*} \otimes S_{f^{*}} x_{1}+f_{1}^{*} \otimes T_{f^{*}} x+V_{f^{*}} \otimes x+W_{f^{*}} \otimes x_{1} \\
& \quad+g^{*} \otimes S_{g^{*}} x_{1}+g_{1}^{*} \otimes T_{g^{*}} x+V_{g^{*}} \otimes x+W_{g^{*}} \otimes x_{1} .
\end{aligned}
$$

Comparing the above equations and applying them to $u-v$,

$$
S_{g^{*}} x_{1}-S_{f^{*}} x_{1}+W_{f^{*}}(u-v) x_{1}+W_{g^{*}}(u-v) x_{1}-W_{f^{*}+g^{*}}(u-v) x_{1} \in L_{0} \cap K_{0}=(0) .
$$

Hence for every $x_{1} \in L_{0}, S_{g^{*}} x_{1}-S_{f^{*}} x_{1}=\lambda_{f^{*}, g^{*}} x_{1}$ for some scalar $\lambda_{f^{*}, g^{*}} \in \mathbb{C}$. The independence of $f^{*}$ and $g^{*}$ implies that $f_{1}^{*}$ and $g_{1}^{*}$ are independent. Similarly, we have that for every $x \in K_{0}, T_{g^{*}} x-T_{f^{*}} x=\mu_{f^{*}, g^{*}} x$ for some scalar $\mu_{f^{*}, g^{*}} \in \mathbb{C}$. We establish the claim.

Now fix a nonzero functional $f_{0}^{*}=f_{01}^{*}+f_{02}^{*} \in K_{p}$, where $f_{01}^{*} \in L_{p}$ and $f_{02}^{*} \in M_{p}$. Set $S=S_{f_{0}^{*}}$ and $T=T_{f_{0}^{*}}$. Let $f^{*}=f_{1}^{*}+f_{2}^{*} \in K_{p}$, where $f_{1}^{*} \in L_{p}$ and $f_{2}^{*} \in M_{p}$. If $f^{*}$ and $f_{0}^{*}$ are linearly independent, then there exist scalars $\lambda_{f^{*}}$ and $\mu_{f^{*}}$ in $\mathbb{C}$ such that $S_{f^{*}} x_{1}-S x_{1}=\lambda_{f^{*}} x_{1}$ for every $x_{1} \in L_{0}$ and $T_{f^{*}} x-T x=\mu_{f^{*}} x$ for every $x \in K_{0}$. Then by (2.5),

$$
\begin{aligned}
\Phi\left(x, f^{*}\right) & =f^{*} \otimes\left(S x_{1}+\lambda_{f^{*}} x_{1}\right)+f_{1}^{*} \otimes\left(T x+\mu_{f^{*}} x\right)+V_{f^{*}} \otimes x+W_{f^{*}} \otimes x_{1} \\
& =f^{*} \otimes S x_{1}+f_{1}^{*} \otimes T x+\left(\lambda_{f^{*}} f^{*}+W_{f^{*}}\right) \otimes x_{1}+\left(\mu_{f^{*}} f_{1}^{*}+V_{f^{*}}\right) \otimes x,
\end{aligned}
$$

for every $x \in K_{0}$. If $f^{*}$ and $f_{0}^{*}$ are linearly dependent, we may assume that $f^{*}=\eta_{f^{*}} f_{0}^{*}$ for some scalar $\eta_{f^{*}} \in \mathbb{C}$. Then $f_{1}^{*}=\eta_{f^{*}} f_{01}^{*}$ and $f_{2}^{*}=\eta_{f^{*}} f_{02}^{*}$. By (2.5),

$$
\begin{aligned}
\Phi\left(x, f^{*}\right) & =\eta_{f^{*}} \Phi\left(x, f_{0}^{*}\right)=\eta_{f^{*}}\left(f_{0}^{*} \otimes S_{f_{0}^{*}} x_{1}+f_{01}^{*} \otimes T_{f_{0}^{*}} x+V_{f_{0}^{*}} \otimes x+W_{f_{0}^{*}} \otimes x_{1}\right) \\
& =f^{*} \otimes S x_{1}+f_{1}^{*} \otimes T x+\eta_{f^{*}} V_{f_{0}^{*}} \otimes x+\eta_{f^{*}} W_{f_{0}^{*}} \otimes x_{1},
\end{aligned}
$$

for every $x \in K_{0}$. It follows from (2.6) and (2.7) that there exist unique functionals $V f^{*}$ and $W f^{*}$ in $X^{*}$ such that $\Phi\left(x, f^{*}\right)=f^{*} \otimes S x_{1}+f_{1}^{*} \otimes T x+V f^{*} \otimes x+W f^{*} \otimes x_{1}$. It is easy to see that the mappings $V, W: K_{p} \rightarrow X^{*}$ are well defined and linear. The proof is complete. 
Proof of Theorem 2.1. Assume that the vector sum $K+L$ is closed. We divide the proof into several claims.

Claim 1. $\delta(I)=\lambda I$ for some scalar $\lambda \in \mathbb{C}$.

For any idempotent $P \in \operatorname{Alg} \mathcal{D}$, since $P(I-P)+(I-P) P=0$, we have $\delta(P)(I-P)+P \delta(I-P)+\delta(I-P) P+(I-P) \delta(P)=0$, which implies that $\delta(I) P=$ $P \delta(I)$. By the proof of [9, Theorem 2.3], we have $\delta(I)=\lambda I$ for some scalar $\lambda \in \mathbb{C}$.

Now define $\tau(A)=\delta(A)-\lambda A$ for every $A \in \operatorname{Alg} \mathcal{D}$. It is easy to see that $\tau$ is Jordan derivable at zero and $\tau(I)=0$. For every $x \in K_{0}$ and every $f^{*} \in K_{p}$, there exist unique vectors $x_{1} \in L_{0}, x_{2} \in M_{0}, f_{1}^{*} \in L_{p}$ and $f_{2}^{*} \in M_{p}$ such that $x=x_{1}+x_{2}$ and $f^{*}=f_{1}^{*}+f_{2}^{*}$. Then $f^{*} \otimes x_{1}-f_{1}^{*} \otimes x \in \operatorname{Alg} \mathcal{D}$ by Theorem 1.2. Define a mapping $\Phi: K_{0} \times K_{p} \rightarrow \operatorname{Alg} \mathcal{D}$ by $\Phi\left(x, f^{*}\right)=\tau\left(f^{*} \otimes x_{1}-f_{1}^{*} \otimes x\right)$. It is easy to see that $\Phi$ is bilinear.

Claim 2. By the above notation, $\Phi\left(x, f^{*}\right)\left(\operatorname{ker}\left(f^{*}\right) \cap \operatorname{ker}\left(f_{1}^{*}\right)\right) \subseteq \operatorname{span}\left\{x, x_{1}\right\}$, for every $x \in K_{0}$ and every $f^{*} \in K_{p}$.

If one of $x$ and $f^{*}$ is 0 , then $\Phi\left(x, f^{*}\right)=0$. We now assume that $x \neq 0$ and $f^{*} \neq 0$.

Case 1. Suppose that $f^{*}\left(x_{1}\right)=m \neq 0$. Then $(1 / m)\left(f^{*} \otimes x_{1}-f_{1}^{*} \otimes x\right)$ is an idempotent in $\operatorname{Alg} \mathcal{D}$. By Lemma 2.2,

$$
\begin{aligned}
\frac{1}{m} \tau\left(f^{*} \otimes x_{1}-f_{1}^{*} \otimes x\right)= & \frac{1}{m^{2}} \tau\left(f^{*} \otimes x_{1}-f_{1}^{*} \otimes x\right)\left(f^{*} \otimes x_{1}-f_{1}^{*} \otimes x\right) \\
& +\frac{1}{m^{2}}\left(f^{*} \otimes x_{1}-f_{1}^{*} \otimes x\right) \tau\left(f^{*} \otimes x_{1}-f_{1}^{*} \otimes x\right) .
\end{aligned}
$$

Applying the above equation to $z$ in $\operatorname{ker}\left(f^{*}\right) \cap \operatorname{ker}\left(f_{1}^{*}\right)$, we obtain $\Phi\left(x, f^{*}\right) z \in$ $\operatorname{span}\left\{x, x_{1}\right\}$.

Case 2. Suppose that $f^{*}\left(x_{1}\right)=0$. Then there exists a vector $y_{1} \in L_{0}$ such that $f^{*}\left(y_{1}\right) \neq$ 0 . Hence there exist unique vectors $y \in K_{0}$ and $y_{2} \in M_{0}$ such that $y_{1}=y-y_{2}$. By Case 1 , for every $z \in \operatorname{ker}\left(f^{*}\right) \cap \operatorname{ker}\left(f_{1}^{*}\right)$,

$$
\begin{aligned}
& \Phi\left(y+x, f^{*}\right) z=k_{1}(y+x)+l_{1}\left(y_{1}+x_{1}\right), \\
& \Phi\left(y-x, f^{*}\right) z=k_{2}(y-x)+l_{2}\left(y_{1}-x_{1}\right),
\end{aligned}
$$

and

$$
\Phi\left(y, f^{*}\right) z=k_{3} y+l_{3} y_{1}
$$

for some scalars $k_{i}, l_{i}(i=1,2,3)$ in $\mathbb{C}$. Comparing the above equations gives

$$
k_{1}(y+x)+l_{1}\left(y_{1}+x_{1}\right)+k_{2}(y-x)+l_{2}\left(y_{1}-x_{1}\right)=2 k_{3} y+2 l_{3} y_{1},
$$

which yields

$$
k_{1}(y+x)+k_{2}(y-x)-2 k_{3} y=2 l_{3} y_{1}-l_{1}\left(y_{1}+x_{1}\right)-l_{2}\left(y_{1}-x_{1}\right) \in K_{0} \cap L_{0}=(0) .
$$

Since $x_{1}$ and $y_{1}$ are linearly independent and $x$ and $y$ are linearly independent, we have $l_{1}=l_{2}=l_{3}$ and $k_{1}=k_{2}=k_{3}$. Hence $\Phi\left(x, f^{*}\right) z=k_{1} x+l_{1} x_{1} \in \operatorname{span}\left\{x, x_{1}\right\}$. 
Claim 3. $\tau$ is a derivation.

By Claim 2 and Lemma 2.4, there exist linear mappings $S: L_{0} \rightarrow L_{0}, T: K_{0} \rightarrow K_{0}$, $V: K_{p} \rightarrow X^{*}$ and $W: K_{p} \rightarrow X^{*}$ such that

$$
\tau\left(f^{*} \otimes x_{1}-f_{1}^{*} \otimes x\right)=f^{*} \otimes S x_{1}+f_{1}^{*} \otimes T x+V f^{*} \otimes x+W f^{*} \otimes x_{1},
$$

for every $x=x_{1}+x_{2} \in K_{0}$ and every $f^{*}=f_{1}^{*}+f_{2}^{*} \in K_{p}$. It follows from Lemma 2.3 that for every $A \in \operatorname{Alg} \mathcal{D}$,

$$
\begin{aligned}
\tau\left(f^{*} \otimes\right. & \left.A x_{1}-f_{1}^{*} \otimes A x+A^{*} f^{*} \otimes x_{1}-A^{*} f_{1}^{*} \otimes x\right) \\
= & f^{*} \otimes \tau(A) x_{1}-f_{1}^{*} \otimes \tau(A) x+A \tau\left(f^{*} \otimes x_{1}-f_{1}^{*} \otimes x\right) \\
& \quad+\tau\left(f^{*} \otimes x_{1}-f_{1}^{*} \otimes x\right) A+\tau(A)^{*} f^{*} \otimes x_{1}-\tau(A)^{*} f_{1}^{*} \otimes x,
\end{aligned}
$$

which according to (2.8) implies that

$$
\begin{aligned}
f^{*} \otimes S A x_{1}+f_{1}^{*} \otimes T A x+V A^{*} f^{*} \otimes x+W A^{*} f^{*} \otimes x_{1} \\
=f^{*} \otimes \tau(A) x_{1}-f_{1}^{*} \otimes \tau(A) x+f^{*} \otimes A S x_{1}+f_{1}^{*} \otimes A T x \\
\quad+A^{*} V f^{*} \otimes x+A^{*} W f^{*} \otimes x_{1}+\tau(A)^{*} f^{*} \otimes x_{1}-\tau(A)^{*} f_{1}^{*} \otimes x .
\end{aligned}
$$

Applying the above equation to $u$ in $X$ such that $f_{1}^{*}(u)=1$, we have that there exists a linear mapping $\mu: \operatorname{Alg} \mathcal{D} \rightarrow \mathbb{C}$ such that

$$
\tau(A) x=A T x-T A x+\mu(A) x,
$$

for every $A \in \operatorname{Alg} \mathcal{D}$ and every $x \in K_{0}$. Hence by (2.9), for $A, B \in \operatorname{Alg} \mathcal{D}$ and $x \in K_{0}$,

$$
\tau(A B) x=\tau(A) B x+A \tau(B) x+\mu(A B) x-\mu(A) B x-\mu(B) A x .
$$

In the following, we show that $\mu(A)=0$ for every $A \in \operatorname{Alg} \mathcal{D}$. Since the vector sum $K+L$ is closed, we have $m=\operatorname{dim} M_{0} \neq 0$ and $n=\operatorname{dim} M_{p} \neq 0$. Hence by Theorem 1.2, there exists a rank-two idempotent in $\operatorname{Alg} \mathcal{D}$. Let $R=u^{*} \otimes v-v^{*} \otimes u$ be a rank-two idempotent in $\operatorname{Alg} \mathcal{D}$, where $u, v \in X$ and $u^{*}, v^{*} \in X^{*}$ are nonzero vectors satisfying $u \in L_{0}, v \in M_{0}, u+v \in K_{0}$ and $u^{*} \in L_{p}, v^{*} \in M_{p}, u^{*}+v^{*} \in K_{p}$. By Lemma 1.3, $u^{*}(v)=$ $-v^{*}(u)=1$. Putting $A=B=R$ and $x=u+v$ in Equation (2.10) gives $\tau(R)(u+v)=$ $\tau(R)(u+v)+R \tau(R)(u+v)-\mu(R)(u+v)$, and Lemma 2.2 implies that $\tau(R)(u+v)=$ $\tau(R)(u+v)+R \tau(R)(u+v)$. Hence $\mu(R)=0$ for every rank-two idempotent $R$ in $\operatorname{Alg} \mathcal{D}$. Now fix a rank-two idempotent $R$ in $\operatorname{Alg} \mathcal{D}$. For every $A \in \operatorname{Alg} \mathcal{D}$, if $u^{*}(A v)=m \neq 0$, then $\mu(A R)=m \mu\left(u^{*} \otimes((1 / m) A v)-v^{*} \otimes((1 / m) A u)\right)=0$; if $u^{*}(A v)=0$, then $\mu(A R)=$ $\mu\left(u^{*} \otimes(v+A v)-v^{*} \otimes(u+A u)\right)-\mu\left(u^{*} \otimes v-v^{*} \otimes u\right)=0$. Hence $\mu(A R)=0$ for every $A \in \operatorname{Alg} \mathcal{D}$. Similarly, $\mu(R A)=0$ for every $A \in \operatorname{Alg} \mathcal{D}$.

Now for every $A \in \operatorname{Alg} \mathcal{D}$, by (2.10),

$$
\tau(A R)(u+v)=\tau(A)(u+v)+A \tau(R)(u+v)-\mu(A)(u+v)
$$

and

$$
\tau(R A)(u+v)=\tau(R) A(u+v)+R \tau(A)(u+v)-\mu(A)(u+v) .
$$


By Lemma 2.3,

$$
\tau(A R+R A)(u+v)=\tau(A)(u+v)+A \tau(R)(u+v)+\tau(R) A(u+v)+R \tau(A)(u+v) .
$$

Hence $\mu(A)=0$ for every $A \in \operatorname{Alg} \mathcal{D}$.

Now for $A, B \in \operatorname{Alg} \mathcal{D}$, by (2.10), we have $\tau(A B) x=\tau(A) B x+A \tau(B) x$ for every $x \in K_{0}$. Since $K_{0}$ is dense in $K$, we have $\tau(A B) x=\tau(A) B x+A \tau(B) x$ for every $x \in K$. Similarly, we have $\tau(A B) x=\tau(A) B x+A \tau(B) x$ for every $x \in L$. Since $K+L=X$, it follows that $\tau$ is a derivation. The proof is complete.

Theorem 2.5. Let $\mathcal{D}$ be a strongly double triangle subspace lattice on $X$ of dimension greater than two and let $\delta: \operatorname{Alg} \mathcal{D} \rightarrow \operatorname{Alg} \mathcal{D}$ be a linear mapping satisfying $\delta(A B+B A)=\delta(A) B+A \delta(B)+\delta(B) A+B \delta(A)$ whenever $A B=0$. Then $\delta$ is a derivation.

To prove Theorem 2.5, we need the following lemma.

LEMMA 2.6. If $\delta$ is a linear mapping from a unital algebra $\mathcal{A}$ into itself satisfying $\delta(A B+B A)=\delta(A) B+A \delta(B)+\delta(B) A+B \delta(A)$ whenever $A B=0$, then for every idempotent $P$ in $\mathcal{A}$ and every $A$ in $\mathcal{A}$, the following statements hold:

(1) $\delta(I) P=P \delta(I)$ and $\delta(P)=\delta(P) P+P \delta(P)-\delta(I) P$;

(2) $\delta(P A+A P)=\delta(P) A+P \delta(A)+\delta(A) P+A \delta(P)-\delta(I) P A-P A \delta(I)$;

(3) $\delta(P A+A P)=\delta(P) A+P \delta(A)+\delta(A) P+A \delta(P)-\delta(I) A P-A P \delta(I)$.

Proof. (1) For every idempotent $P \in \mathcal{A}$, it follows from $P(I-P)=0$ that $\delta(P)(I-P)+P \delta(I-P)+\delta(I-P) P+(I-P) \delta(P)=0$. This implies that $2 \delta(P)=$ $2 \delta(P) P+2 P \delta(P)-\delta(I) P-P \delta(I)$. Multiplying the above equation from the left and right by $P$, respectively, we have $P \delta(I)=\delta(I) P$, which yields $\delta(P)=\delta(P) P+$ $P \delta(P)-\delta(I) P$.

(2) For every idempotent $P \in \mathcal{A}$ and every $A \in \mathcal{A}$, since $P(I-P) A=(I-P) P A=0$, we have

$\delta(P(I-P) A+(I-P) A P)=\delta(P)(I-P) A+P \delta((I-P) A)+\delta((I-P) A) P+(I-P) A \delta(P)$

and

$$
\delta((I-P) P A+P A(I-P))=\delta(I-P) P A+(I-P) \delta(P A)+\delta(P A)(I-P)+P A \delta(I-P) .
$$

Comparing the above equations, we arrive at $\delta(P A+A P)=\delta(P) A+P \delta(A)+\delta(A) P+$ $A \delta(P)-\delta(I) P A-P A \delta(I)$.

(3) Since $A P(I-P)=A(I-P) P=0$, we similarly have that $\delta(P A+A P)=\delta(P) A+$ $P \delta(A)+\delta(A) P+A \delta(P)-\delta(I) A P-A P \delta(I)$.

Proof of Theorem 2.5. We claim that $\delta(I)=0$. Similar to the proof of [9, Theorem 2.3], we have $\delta(I)=\lambda I$ for some scalar $\lambda \in \mathbb{C}$. Suppose that $\lambda \neq 0$. Then by Lemma 2.6(2) and (3), $A P=P A$ for every idempotent $P$ in $A \lg \mathcal{D}$ and every $A$ in Alg $\mathcal{D}$. By the proof of [9, Theorem 2.3] again, we have that $A=\mu(A) I$ for some scalar $\mu(A) \in \mathbb{C}$. That is, for every $A \in \operatorname{Alg} \mathcal{D}$, the range of $A$ is $X$ or 0 . However, since $\mathcal{D}$ is 
strongly double triangle subspace lattice, $\operatorname{Alg} \mathcal{D}$ contains a rank-two operator. This is a contradiction. Hence $\delta(I)=0$. Then by the proof of Theorem 2.1, we may show that $\delta$ is a derivation.

Remark 2.7. In Theorem 2.5, if $\operatorname{dim} X=2, \delta$ may not be a derivation since $A \lg \mathcal{D}=\mathbb{C} I$.

\section{References}

[1] M. Chebotar, W. Ke and P. Lee, 'Maps characterized by action on zero products', Pacific J. Math. 216 (2004), 217-228.

[2] P. Halmos, 'Reflexive lattices of subspaces', J. Lond. Math. Soc. 4(2) (1971), 257-263.

[3] M. Jiao and J. Hou, 'Additive maps derivable or Jordan derivable at zero point on nest algebras', Linear Algebra Appl. 432 (2010), 2984-2994.

[4] W. Jing, S. Lu and P. Li, 'Characterisations of derivations on some operator algebras', Bull. Aust. Math. Soc. 66 (2002), 227-232.

[5] R. Kadison and J. Ringrose, Fundamentals of the Theory of Operator Algebras, Vols. I and II (Academic Press, London, 1983, 1986).

[6] M. Lambrou and W. E. Longstaff, 'Finite rank operators leaving double triangles invariant', J. Lond. Math. Soc. 45 (1992), 153-168.

[7] W. E. Longstaff, 'Strongly reflexive lattices', J. Lond. Math. Soc. 11 (1975), 491-498.

[8] W. E. Longstaff, 'Non-reflexive double triangles', J. Aust. Math. Soc. 35 (1983), 349-356.

[9] Y. Pang and W. Yang, 'Derivations and local derivations on strongly double triangle subspace lattice algebras', Linear Multilinear Algebra 58 (2010), 855-862.

[10] S. Zhao and J. Zhu, 'Jordan all-derivable points in the algebra of all upper triangular matrices', Linear Algebra Appl. 433 (2010), 1922-1938.

YUN-HE CHEN, Department of Mathematics, East China University of Science and Technology, Shanghai 200237, PR China e-mail: heyunchen@hotmail.com

JIAN-KUI LI, Department of Mathematics, East China University of Science and Technology, Shanghai 200237, PR China e-mail: jiankuili@yahoo.com 